\title{
Improved efficiency of selective photoionization of palladium isotopes via autoionizing Rydberg states
}

\author{
Clayton R. Locke ${ }^{1}$ Tohru Kobayashi ${ }^{1} \cdot$ Katsumi Midorikawa $^{1}$
}

Received: 7 October 2016 / Accepted: 19 December 2016 / Published online: 28 December 2016

(c) The Author(s) 2016. This article is published with open access at Springerlink.com

\begin{abstract}
Odd-mass-selective ionization of palladium for purposes of resource recycling and management of long-lived fission products can be achieved by exploiting transition selection rules in a well-established three-step excitation process. In this conventional scheme, circularly polarized lasers of the same handedness excite isotopes via two intermediate ${ }^{2} \mathrm{D}_{5 / 2}$ core states, and a third laser is then used for ionization via autoionizing Rydberg states. We propose an alternative excitation scheme via intermediate ${ }^{2} \mathrm{D}_{3 / 2}$ core states before the autoionizing Rydberg state, improving ionization efficiency by over 130 times. We confirm high selectivity and measure odd-mass isotopes of $>99.7(3) \%$ of the total ionized product. We have identified and measured the relative ionization efficiency of the series of Rydberg states that converge to upper ionization limit of the $4 d^{9}\left({ }^{2} \mathrm{D}_{3 / 2}\right)$ level, and identify the most efficient excitation is via the Rydberg state at $67668.18(10) \mathrm{cm}^{-1}$.
\end{abstract}

\section{Introduction}

Palladium is one of the high-value platinum metals present in nuclear power plant radioactive waste, occurring in quantities comparable to that available from natural reserves [1]; however, they are presently unable to be utilized due to the co-presence of the long-lived radioactive isotopes ${ }^{107} \mathrm{Pd}(17 \%)$. To date, techniques to selectively remove this radioactive isotope have not been established on a commercial scale. As the isotope shifts in palladium

Clayton R. Locke

clayton.locke@riken.jp

1 RIKEN Center for Advanced Photonics, 2-1 Hirosawa, Wako, Saitama 351-0198, Japan are only approximately $50 \mathrm{MHz}$ [2], it is not feasible to selectively ionize isotopes using narrow linewidth lasers; however, two effective techniques exist for selective ionization. The first is based on a proposal by Balling and Wright [1] using circularly polarized lasers, in which only odd-mass number isotopes having nonzero nuclear spin and thus having hyperfine structure are able to be excited due to optical transition selection rules. The feasibility of this scheme as applied to platinum group metals is reported by Chen [3], implemented by Yamaguchi and Sasao [4, 5], and further developed in our previous paper [6]. The second technique, resonant ionization mass spectrometry (RIMS) $[7,8]$, obtains selectivity of ionized isotopes via a narrow slit and accelerating fields, such as the RISIKO separator system as applied to palladium [9]. Comparison between the efficiencies and costs of both techniques are essential before commercial-scale projects can be undertaken.

We have developed our apparatus employing the first technique, that is, exploiting transition rules for selective excitation [10]. Resonant excitation to autoionizing states is a promising means to obtain efficient ionization after such mass-selective excitation. Autoionizing Rydberg states come about due a characteristic in the spin-orbit interaction of $\mathrm{Pd}$, where the ion Pd II has two ground states of different ion core configurations, namely $4 d^{9}\left({ }^{2} \mathrm{D}_{5 / 2}\right)$ at $67241.3(8) \mathrm{cm}^{-1}$ and $4 d^{9}\left({ }^{2} \mathrm{D}_{3 / 2}\right)$ at $70779.8(8) \mathrm{cm}^{-1}$ [11]. Any states existing between the two ground states are autoionizing, and the ion yield is over an order of magnitude higher than excitation to the ionization continuum [12].

The conventional Pd odd-mass selectivity excitation scheme excites via intermediate states having a ${ }^{2} D_{5 / 2}$ core [3-6], as shown as configuration (a) in Fig. 1. For this scheme, selectivity is obtained via (0-1-0) transition rules, that is, the first level is $J=0$, the first intermediate state is $J=1$, and the second intermediate state is $J=0$. If 
Fig. 1 Two-step selective excitation scheme, with a third step to autoionizing Rydberg states; a the conventional (0-1-0) scheme using circularly polarized light for selectivity; b the proposed (0-1-1) scheme using linearly polarized light for selectivity. The frequency of the third excitation laser $(\omega 3)$ is swept over the ranges indicated to map autoionizing Rydberg states converging to Pd II $4 d^{9}\left({ }^{2} \mathrm{D}_{3 / 2}\right)$. Energies and level designations are from NIST Atomic Spectra Database [11]

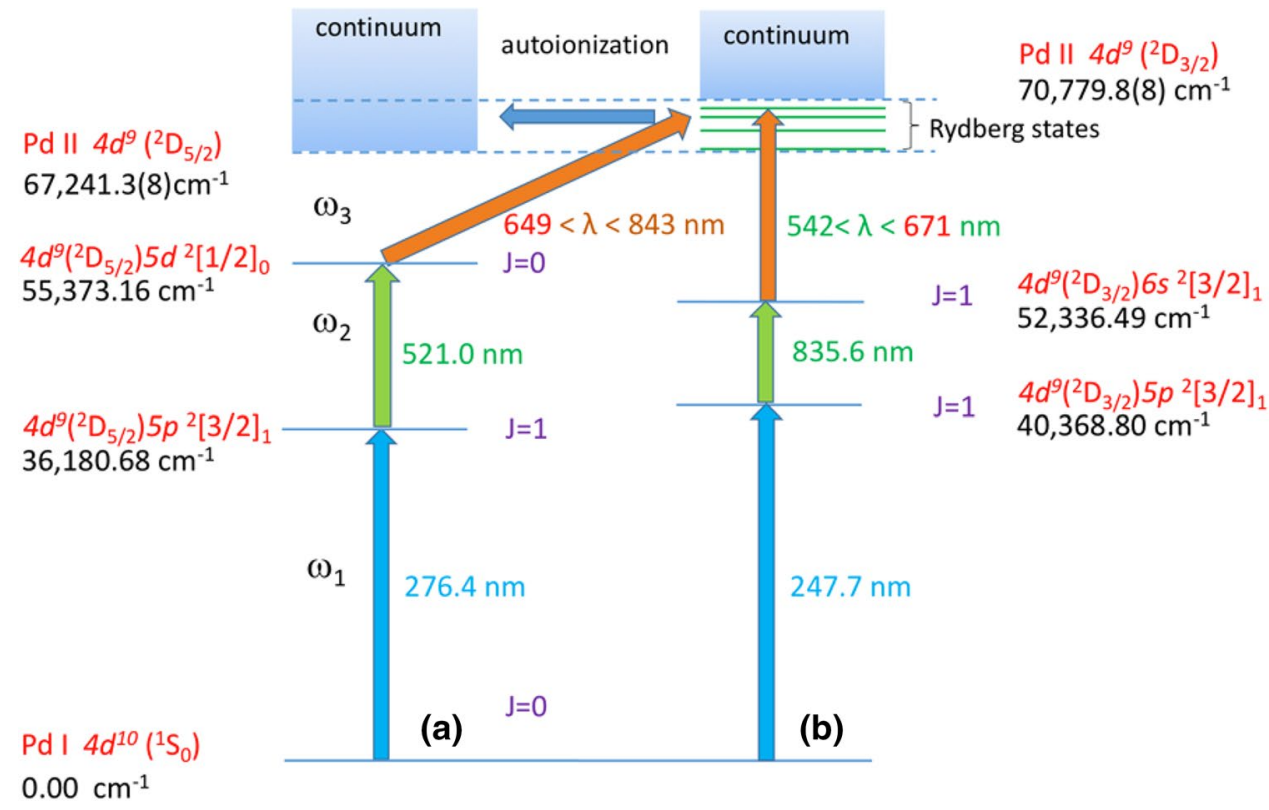

The first dye laser at $495.4 \mathrm{~nm}$ is frequency doubled in a BBO crystal to produce the first excitation wavelength $\left(\lambda_{1}\right)$ of $247.7 \mathrm{~nm}$, exciting Pd atoms from the ground state into the $4 d^{9}\left({ }^{2} \mathrm{D}_{3 / 2}\right) 5 p^{2}[3 / 2]_{1}$ state, which is optimally tuned by maximizing fluorescence to the $4 d^{9}\left({ }^{2} \mathrm{D}_{3 / 2}\right) 5 s^{2}[3 / 2]_{1}$ state, detected at $330.3 \mathrm{~nm}$ by a photomultiplier tube (Hamamatsu R955) through a monochromator (Jasco: CT-25C). The second laser at $835.6 \mathrm{~nm}$ excites Pd atoms from $4 d^{9}\left({ }^{2} \mathrm{D}_{3 / 2}\right) 5 p^{2}[3 / 2]_{1}$ to $4 d^{9}\left({ }^{2} \mathrm{D}_{3 / 2}\right) 6 s^{2}[3 / 2]_{1}$, tuned by maximizing the decay emission to $4 d^{9}\left({ }^{2} \mathrm{D}_{3 / 2}\right) 5 p{ }^{2}[5 / 2]_{2}$ at $739.4 \mathrm{~nm}$. The third laser is inserted colinear to the second laser with a dichroic mirror, counter-propagating to the first laser, and is swept over the entire range between the ionic ground states of Pd II.

Photoions are produced in a field-free region to reduce frequency shift effects and are subsequently horizontally repelled by a set of three electrodes charged with a timedelayed high-voltage pulse (switched on $1.8 \mu \mathrm{s}$ after photoionization) in a Wiley-McLaren configuration [13] in order to separate mass isotopes in a $1-\mathrm{m}$ time-of-flight mass spectrometer, with mass resolution $(\mathrm{m} / \Delta \mathrm{m})$ of 600 . The relative amounts of Pd isotopes are detected using a 2-stack microchannel plate (Hamamatsu F4655-11). Statistical fluctuations in the observed signal are averaged over several hundred laser shots on a $500-\mathrm{MHz}$ storage oscilloscope. Ion current is calculated from the area under the voltage-time curve, via the input impedance of the oscilloscope.

The ion current is observed as a function of $\omega 3$ to determine resonant transition frequencies of autoionizing Rydberg levels. Intensity variations in the third laser over the range of the frequency scan, measured by sampling a portion of the beam using a photodetector, are normalized 


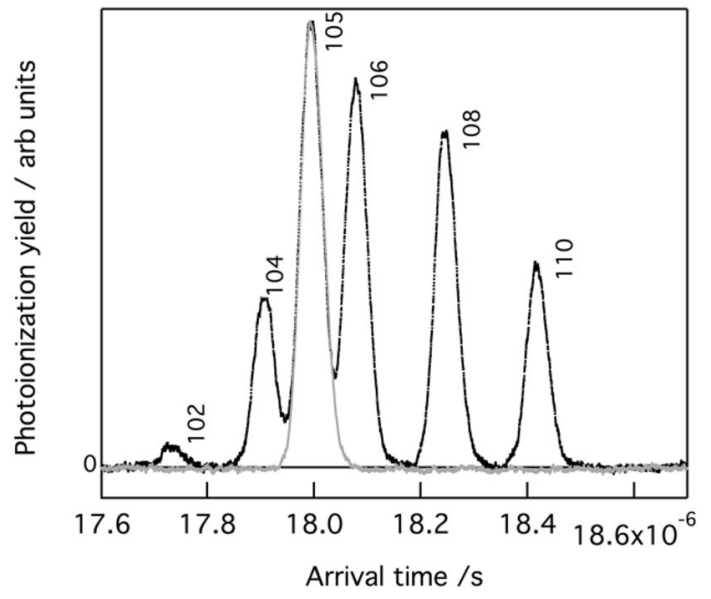

Fig. 2 Observed isotopes of Pd II; - polarization axes of the two (linearly polarized) excitation lasers are not aligned and all isotopes of Pd are observed $\left({ }^{102} \mathrm{Pd}: 1.1 \%,{ }^{104} \mathrm{Pd}: 10.7 \%,{ }^{105} \mathrm{Pd}: 28.9 \%,{ }^{106} \mathrm{Pd}\right.$ : $\left.25.2 \%,{ }^{108} \mathrm{Pd}: 21.5 \%,{ }^{110} \mathrm{Pd}: 12.8 \%\right)$; - polarization axes are aligned, only odd-mass number isotopes are excited and subsequently ionized, with $<0.3 \%$ of even-mass number isotopes ionized

in post-processing to determine relative transition intensities. Four different dye solutions are required to span the entire frequency range, so several overlapping peaks are selected and compared to ensure congruous intensity scaling between each dye.

\section{Results and discussion}

Selectivity of odd-mass number isotopes using our proposed ${ }^{2} \mathrm{D}_{3 / 2}$ core intermediate states scheme is shown in Fig. 2. In these demonstrative experiments, we use natural samples in which isotope ${ }^{107} \mathrm{Pd}$ (the radioactive isotope of interest in selectivity schemes) is not present. However, as ${ }^{105} \mathrm{Pd}$ and ${ }^{107} \mathrm{Pd}$ have the same nuclear spin $(I=5 / 2)$, both isotopes are expected to behave identically in the separation process. For comparison, we show the observed signal when the first two excitation lasers do not have their polarization axes aligned, and all isotopes are observed in ratios that are expected in natural abundance [14]. When the polarization axes of the excitations lasers are aligned, complete selectivity is achieved, with only odd-mass number isotopes ionized. It is of note that the selection process does not alter the amount of odd-mass number isotopes that are ionized in comparison with when all isotopes are ionized.

One important distinction between this transition-rule mass-selective method and that using ion mass selection (RIMS) is that the former has strict limitations on the fluence of the first laser, in that it must be below the threshold for two-photon ionization, as described previously [6].
Conversely, an advantage to the former is that only isotopes of interest are excited and thus overall yield is not limited by ion current, whereas in the RIMS method ion-ion Coulombic repulsion imposes a limit on selectivity with increased ion current.

We have investigated the autoionizing Rydberg series by scanning the third laser $(\omega 3)$ over the entire range between the two ionization potentials to find the most efficient transition, as shown in Fig. 3. Peak positions are calculated by fitting a Lorentzian to experimental data points, with peak center uncertainty of $\pm 0.1 \mathrm{~cm}^{-1}$. Spectral lines are listed in Table 2.

The most efficient autoionization state was found to be at $67668.39(10) \mathrm{cm}^{-1}$, corresponding to a third-step laser wavelength of $652.244(5) \mathrm{nm}$. The proposed ${ }^{2} \mathrm{D}_{3 / 2}$ core scheme with autoionization to this Rydberg level has an ion current over 130 times larger than the conventional ${ }^{2} \mathrm{D}_{5 / 2}$ core scheme $[3,4,15-17]$ when $\omega 3$ is tuned to the corresponding maximal ion current Rydberg level of this scheme occurring at $69055.51(30) \mathrm{cm}^{-1}$. Comparison of ion current between both schemes as a function of fluence of $\omega 3$ is shown in Fig. 4.

Peaks due to ionization via the sequence $(\omega 1 \rightarrow \omega 3 \rightarrow \omega 2)$ have been identified at $(67340.855,70106.338,70163.113$, 70375.986, 70416.395, 70523.647, 70649.190) $\mathrm{cm}^{-1}$, where experimentally measured peaks differ from the NIST spectra database [11] by $<0.20 \mathrm{~cm}^{-1}$. Although ion current is substantially lower (transitions are to the continuum rather than autoionizing Rydberg states), it is important that these peaks are identified as ionization is via intermediate states equally allowed for both odd and even mass isotopes.

The energies of the Rydberg states $E(n)$ can be approximated by the extended Ritz formula [18, 19]:

$E(n)=$ I.P. $-\frac{R_{P d}}{[n-\delta(n)]^{2}}$

where

$\delta(n)=\delta_{0}+\frac{\delta_{2}}{\left(n-\delta_{0}\right)^{2}}$

where I.P. is the ionization energy of Pd I, $R_{P d}$ is the masscorrected Rydberg constant of Pd I $\left(109736.75 \mathrm{~cm}^{-1}\right)$, and both $\delta_{0}$ and $\delta_{2}$ are the energy-dependent quantum defects that account for the shielding of the nucleus by the core electrons.

For assignment of the energy levels, we use the $J_{c} K$ coupling scheme [20] which is appropriate for Pd I. Selection rules for the transition from the $4 d^{9} 6 s$ state indicate eight Rydberg series are expected, five of which are autoionizing: $4 d^{9}\left({ }^{2} \mathrm{D}_{3 / 2}\right) n p[1 / 2]_{0}, 4 d^{9}\left({ }^{2} \mathrm{D}_{3 / 2}\right) n p[3 / 2]_{1}, 4 d^{9}\left({ }^{2} \mathrm{D}_{3 /}\right.$ 
Fig. 3 High-resolution Rydberg spectrum observed from the intermediate state Pd I $4 d^{9}\left({ }^{2} \mathrm{D}_{3 / 2}\right) 6 s^{2}[3 / 2]_{1} ;$ O ionic ground state of Pd II $4 d^{9}\left({ }^{2} \mathrm{D}_{5 / 2}\right)$ having value of $67241.3 \mathrm{~cm}^{-1}$; $\times$ ionic ground state of $\mathrm{Pd}$ II $4 d^{9}\left({ }^{2} \mathrm{D}_{3 / 2}\right)$ having value of $70780.40 \mathrm{~cm}^{-1}$. Guidelines and integers indicate principal quantum numbers of Rydberg series. Arrows indicate peaks arising from the sequence $(\omega 1 \rightarrow \omega 3 \rightarrow \omega 2)$
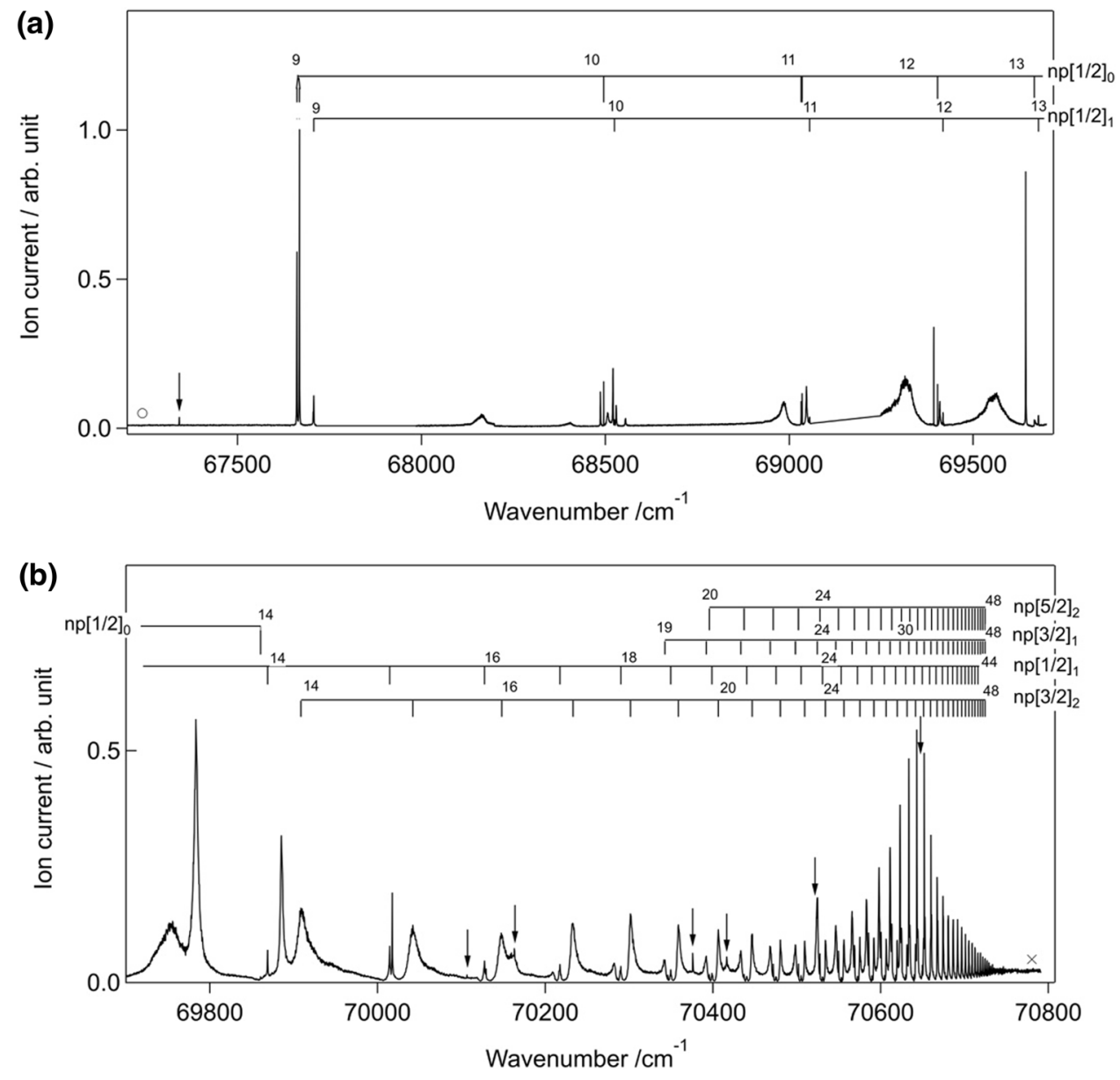

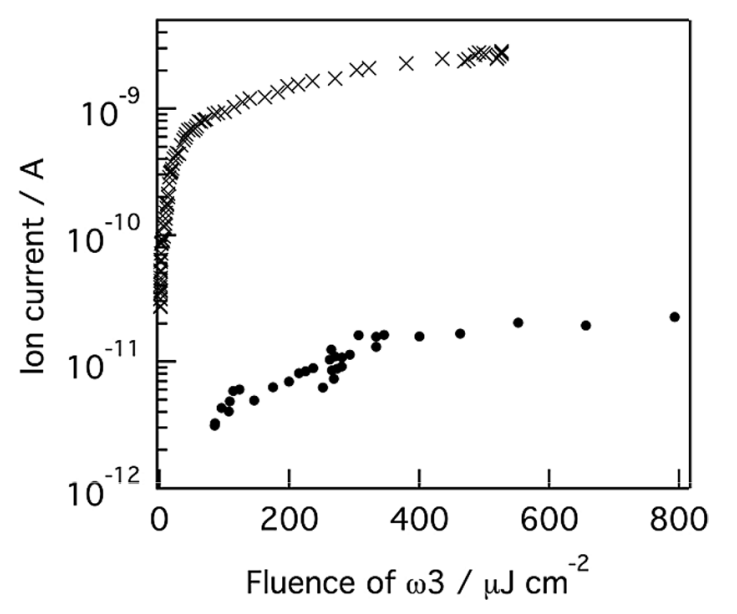

Fig. 4 Observed ion current in the two different odd-mass isotope selectivity schemes. - using the conventional ${ }^{2} \mathrm{D}_{5 / 2}$ core scheme; $x$ using the proposed ${ }^{2} \mathrm{D}_{3 / 2}$ core scheme
Table 1 Fitting coefficients ( \pm 1 standard deviation) to the extended Ritz formula; $\delta_{0}$ and $\delta_{2}$ are the energy-dependent quantum defects

\begin{tabular}{lll}
\hline & $\delta_{0}$ & $\delta_{2}$ \\
\hline $4 d^{9}\left({ }^{2} \mathrm{D}_{3 / 2}\right) n p[3 / 2]_{1}$ & $2.73(2)$ & $-143(5)$ \\
$4 d^{9}\left({ }^{2} \mathrm{D}_{3 / 2}\right) n p[5 / 2]_{2}$ & $2.66(6)$ & $-163(19)$ \\
$4 d^{9}\left({ }^{2} \mathrm{D}_{3 / 2}\right) n p[1 / 2]_{1}$ & $3.029(2)$ & $-0.17(6)$ \\
$4 d^{9}\left({ }^{2} \mathrm{D}_{3 / 2}\right) n p[3 / 2]_{2}$ & $2.926(5)$ & $-17.3(8)$ \\
$4 d^{9}\left({ }^{2} \mathrm{D}_{3 / 2}\right) n p[1 / 2]_{0}$ & $3.082(1)$ & $-0.56(4)$ \\
\hline
\end{tabular}

$\left.{ }_{2}\right) n p[1 / 2]_{1}, 4 d^{9}\left({ }^{2} \mathrm{D}_{3 / 2}\right) n p[5 / 2]_{2}$, and $4 d^{9}\left({ }^{2} \mathrm{D}_{3 / 2}\right) n p[3 / 2]_{2}$. Of these, only $4 d^{9}\left({ }^{2} \mathrm{D}_{3 / 2}\right) n p[1 / 2]_{1}$ and $4 d^{9}\left({ }^{2} \mathrm{D}_{3 / 2}\right) n p[3 / 2]_{1}$ are in common with those observed when exciting Pd I electrons from a $d$ state: This can be either via direct excitation from the $4 d^{10}$ ground state with VUV radiation, as is reported in Baig et al. [21] in which the $n p$ Rydberg series $(n=8-17$, 


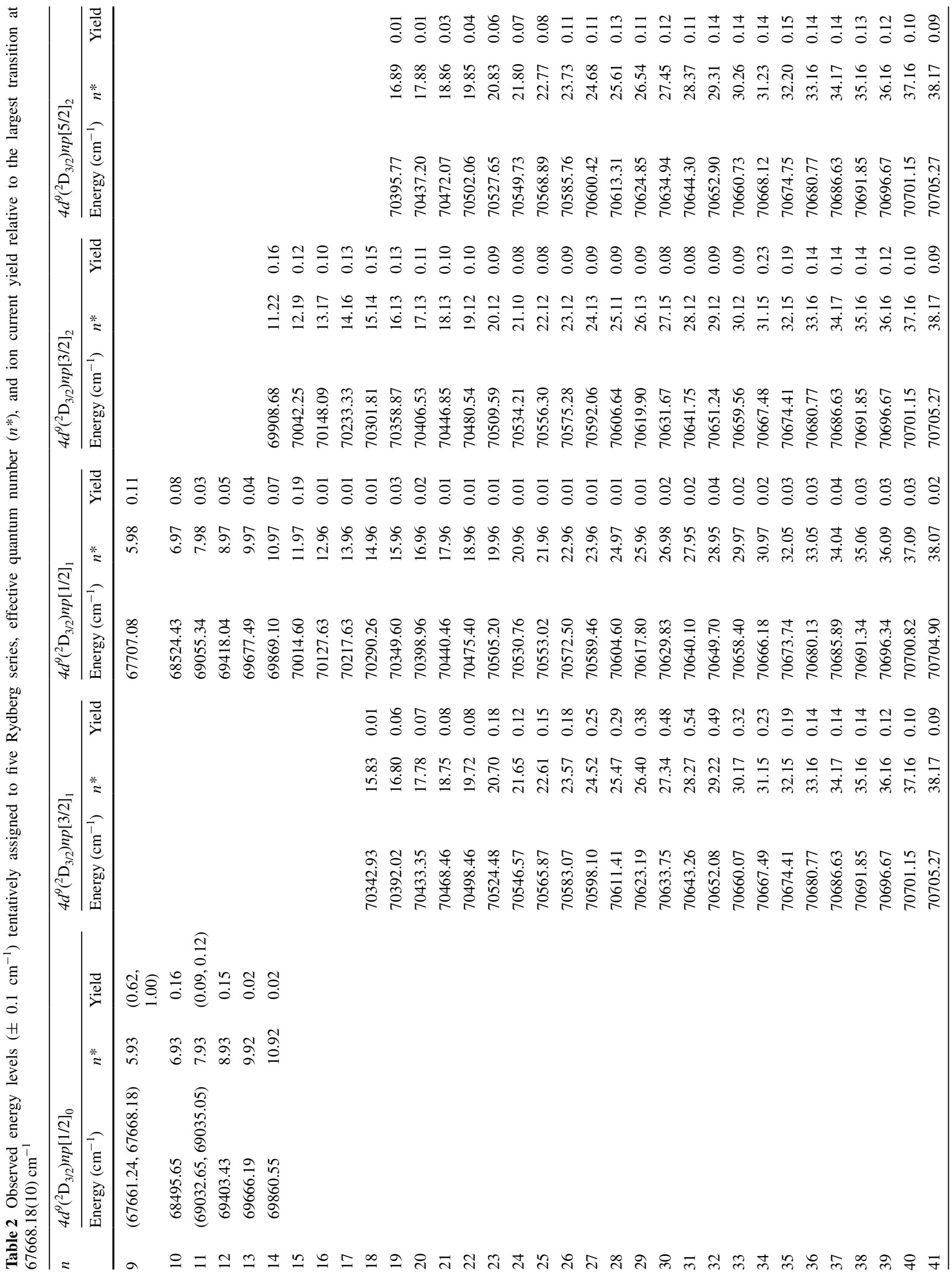




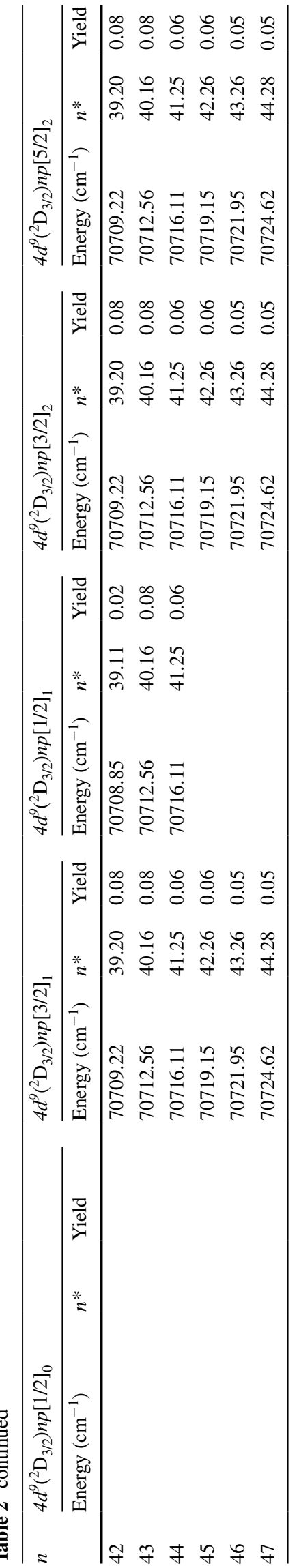

$\delta=3.02$ ) were observed, or alternatively, these two series are also observed in our earlier study from the $4 d^{9} 5 d$ intermediate state [22]. The co-incidence of the two $n p$ series in [22] allows us to assign two of the five observed series in this work.

We calculate the principal quantum number by extrapolating to lower-lying levels and matching to identified levels [11], and tentatively assign the observed series. The lowest member of the series assigned to $4 d^{9}\left({ }^{2} \mathrm{D}_{3 / 2}\right) n p[3 / 2]_{2}$ has the largest ion current and exhibits fine-structure splitting about the predicted position of $67664.30 \mathrm{~cm}^{-1}$, suggesting interaction with some local perturbation. Similarly, we note energy level splitting around $(68,500,69,050,69,400$, and $69,700) \mathrm{cm}^{-1}$, and we observe distorted profiles in a series of broad linewidth spectral lines that suggest a strong interaction with a resonance around $69,700 \mathrm{~cm}^{-1}$. However, further analysis on the origin of these interactions is difficult without knowledge of spectroscopic data [11] on two-electron excited states of palladium.

The energy of Pd II $4 d\left({ }^{2} \mathrm{D}_{3 / 2}\right)$ is determined to be $70780.6(1) \mathrm{cm}^{-1}$ from least squares fitting of Eq. 1 to experimentally observed data. This ionization potential can be compared to the range reported in literature, which are 70779.8(8) $\mathrm{cm}^{-1}$ [21], 70780.9(10) $\mathrm{cm}^{-1}$ [12], and $70780.38(8) \mathrm{cm}^{-1}$ [22]. By least squares fitting, we also determine the quantum defects of the observed Rydberg series, as shown in Table 1. Residuals of the fits to Eq. 1 are shown in Fig. 5.

\section{Conclusion}

We have demonstrated an increase in efficiency of over 130 times in odd-mass-selective ionization using a newly proposed three-step excitation scheme when compared to the current art, with a odd-mass isotope selectivity of 99.7(3)\%. This new excitation scheme has as the first two steps parallel linearly polarized lasers instead of circularly polarized lasers, and has greater yield efficiency as these intermediate states share the same core state as the third step to autoionizing Rydberg states. We have observed five autoionizing Rydberg series by scanning the thirdstep laser over the entire range between the two ionization potentials of palladium and by using fits to the extended Ritz formula have derived the energy of Pd II $4 d\left({ }^{2} \mathrm{D}_{3 / 2}\right)$ to be 70780.6(1) $\mathrm{cm}^{-1}$ and determined the quantum defects of these Rydberg series. We have made the first report of relative transition efficiencies to these autoionizing Rydberg states from the $6 s$ intermediate state, a critical step toward development of practical implementation of even- to oddmass separation schemes. The most efficient autoionization state is at $67668.18(10) \mathrm{cm}^{-1}$, corresponding to a thirdstep laser wavelength of $652.244(5) \mathrm{nm}$. The second-most 
Fig. 5 Residuals of least squares fit to extended Ritz formula of observed peaks in ion current, tentatively assigned to Rydberg series. Solid line extended Ritz formula of Eq. (1); $4 d^{9}\left({ }^{2} \mathrm{D}_{3 / 2}\right) n p[3 / 2]_{1}$; - $4 d^{9}\left({ }^{2} \mathrm{D}_{3 / 2}\right) n p[5 / 2]_{2}$ $4 \mathrm{~d}^{9}\left({ }^{2} \mathrm{D}_{3 / 2}\right) n p[1 / 2]_{1} ; \bigcirc$ $4 d^{9}\left({ }^{2} \mathrm{D}_{3 / 2}\right) n p[3 / 2]_{2} ;+4 d^{9}\left({ }^{2} \mathrm{D}_{3 / 2}\right.$ ) $n[1 / 2]_{0}$

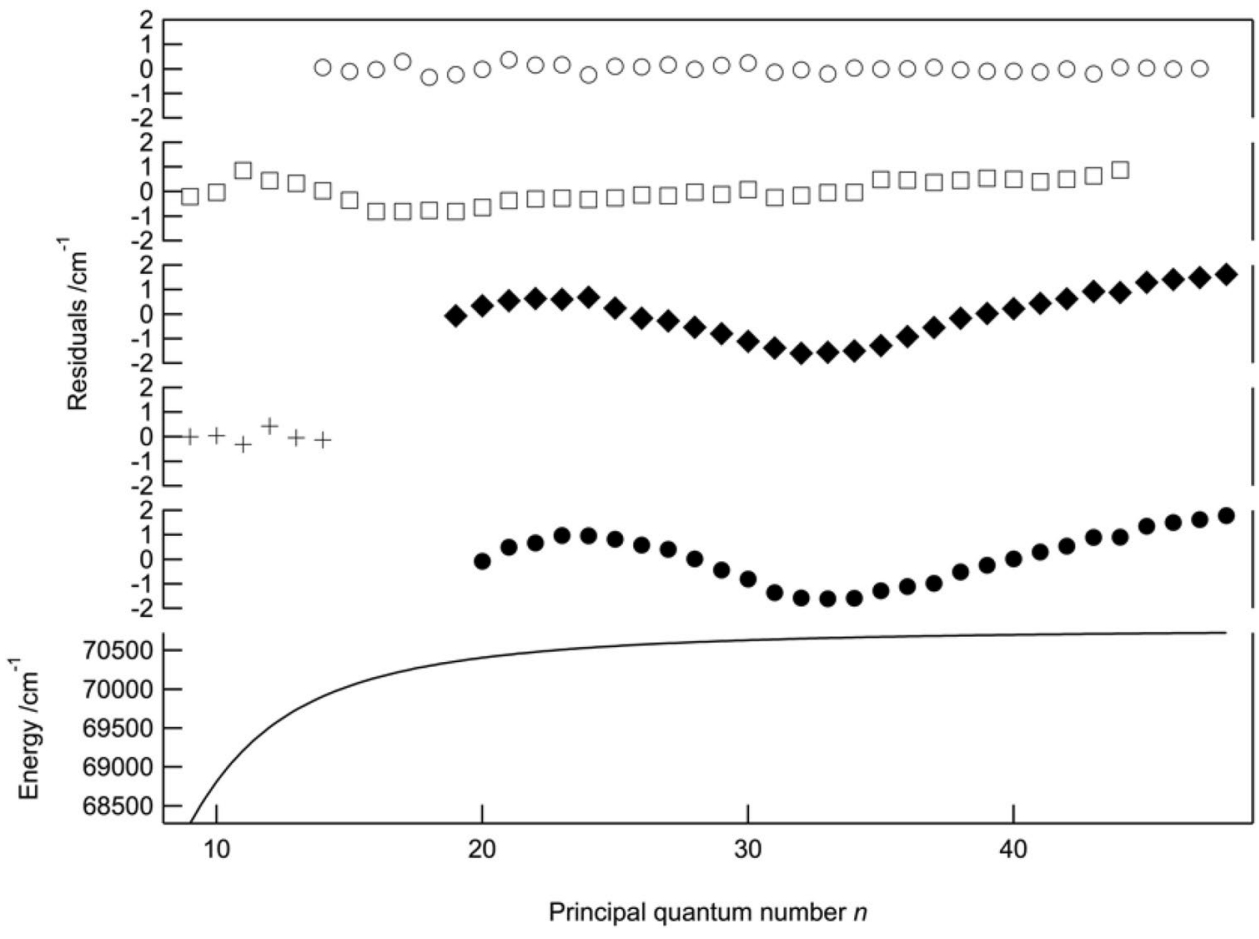

efficient state (having a yield of $86 \%$ of the most efficient) is at $69642.97(10) \mathrm{cm}^{-1}$, corresponding to a third-step laser wavelength of $577.818(5) \mathrm{nm}$.

Acknowledgement This work was funded by the ImPACT Program of the Council for Science, Technology and Innovation (Cabinet Office, Government of Japan).

Open Access This article is distributed under the terms of the Creative Commons Attribution 4.0 International License (http://creativecommons.org/licenses/by/4.0/), which permits unrestricted use, distribution, and reproduction in any medium, provided you give appropriate credit to the original author(s) and the source, provide a link to the Creative Commons license, and indicate if changes were made.

\section{References}

1. K. Naito, T. Matsui, Y. Tanaka, Recovery of noble metals from insoluble residue of spent fuel. J. Nucl. Sci. Technol. 23, 540549 (1986). doi:10.1080/18811248.1986.9735017

2. E. Van Duijn, S. Witte, R. Zinkstok, W. Hogervorst, Hyperfine structure and isotope shift measurements on $4 \mathrm{~d} 101 \mathrm{~s} 0 \rightarrow 4 \mathrm{~d} 95 \mathrm{p}$ $\mathrm{J}=1$ transitions in Pd I using deep-Uv Cw laser spectroscopy. Eur. Phys. J. D 19, 25-29 (2002). doi:10.1140/epjd/e20020051

3. H.L. Chen, Laser Cleanup of Pt Group Metals. UCID-18837 (1980). doi:10.2172/6748034

4. H. Yamaguchi, N. Sasao, The separation of Pd-107 isotope by laser, in Proceedings of the International Symposium on Advanced Nuclear Energy Research (1989)

5. H. Yamaguchi, [cited March 2016]; Available from: jolissrchinter.tokai-sc.jaea.go.jp/pdfdata/PNC-TN8410-95-077.pdf (1995)
6. C.R. Locke, T. Kobayashi, T. Nakajima, K. Midorikawa, Application of an orthogonally polarized laser scheme for selective photoionization of palladium isotopes. Appl. Phys. B 122, 246 (2016). doi:10.1007/s00340-016-6508-7

7. L.P. Smith, W. Parkins, A. Forrester, On the separation of isotopes in quantity by electromagnetic means. Phys. Rev. 72, 989 (1947)

8. M. Payne, L. Deng, N. Thonnard, Applications of resonance ionization mass spectrometry. Rev. Sci. Instrum. 65, 2433-2459 (1994). doi:10.1063/1.1144702

9. T. Kron, Y. Liu, S. Richter, F. Schneider, K. Wendt, High efficiency resonance ionization of palladium with ti: sapphire lasers. J. Phys. B: Atomic Mol. Opt. Phys. (2016). doi:10.1088/0953-4075/49/18/185003

10. V. Radojevic, W. Johnson, Theoretical study of photoabsorption for the palladium 4d subshell. J. Phys. B: Atomic Mol. Phys. 16, 177 (1983). doi:10.1088/0022-3700/16/2/007

11. A. Kramida, Y. Ralchenko, J. Reader, NIST ASD Team, Nist Atomic Spectra Database (Version 5.3) (National Institute of Standards and Technology, Gaithersburg, 2015)

12. C. Callender, P.A. Hackett, D.M. Rayner, First-ionization potential of ruthenium, rhodium, and palladium by doubleresonance ionization spectroscopy. JOSA B 5, 614-618 (1988). doi:10.1364/JOSAB.5.000614

13. W. Wiley, I.H. Mclaren, Time of flight mass spectrometer with improved resolution. Rev. Sci. Instrum. 26, 1150-1157 (1955). doi:10.1063/1.1715212

14. M. Berglund, M.E. Wieser, Isotopic compositions of the elements 2009 (Iupac Technical Report). Pure Appl. Chem. 83, 397-410 (2011)

15. A.N. Tkachev, S.I. Yakovlenko, On laser rare-isotope separation. Quant. Elect. 33, 581 (2003)

16. V.I. Derzhiev et al., Two-step photoionisation of palladium. Quant. Elect. 32, 619 (2002). doi:10.1070/ QE2002v032n07ABEH002257

17. N. Sasao, H. Yamaguchi, Laser Isotope Separation Apparatus. 1992, US Patent US005110562A 
18. W. Ritz, Magnetische atomfelder und serienspektren. Ann. Phys. 330, 660-696 (1908). doi:10.1002/andp.19083300403

19. D.R. Hartree, The wave mechanics of an atom with a NonCoulomb central field. Math. Proc. Camb. Phil. Soc. 24, 89-110 (1928)

20. R.D. Cowan, The Theory of Atomic Structure and Spectra (Univ of California Press, Berkeley, 1981)
21. M. Baig, A. Rashid, Z. Iqbal, J. Hormes, High resolution absorption spectrum of palladium in the $4 \mathrm{~d}$ subshell excitation region. J. Phys. B. 24, 2295 (1991). doi:10.1088/0953-4075/24/9/009

22. T. Kobayashi, C.R. Locke, K. Midorikawa, Spectroscopic investigation of autoionizing Rydberg states of palladium accessible after odd-mass-selective laser excitation. Jpn. J. Appl. Phys. 56, 010302 (2016). doi:10.7567/JJAP.56.010302 